\title{
A new instrument for measuring optimism and pessimism: Test-retest reliability and relations with happiness and religious commitment
}

\author{
WILLIAM N. DEMBER and JUDITH BROOKS \\ University of Cincinnati, Cincinnati, Ohio
}

\begin{abstract}
The main purpose of the present study was to assess the test-retest reliability of a newly devised instrument for measuring optimism and pessimism. In addition, correlations were obtained between the optimism and pessimism scores and two measures of happiness as well as with subjects' responses to single-item questions about the likelihood of a nuclear encounter and about their religious and political commitment and philosophy of life. The instruments were administered to 106 introductory psychology students on two occasions separated by a 2 -week interval. The test-retest reliability for the optimism scale was .75; for pessimism, .84 . Both optimism and pessimism were correlated, on both administrations, with both happiness measures in the high $.50 \mathrm{~s}$ to low $.60 \mathrm{~s}$. Of the remaining items, the strongest relation was between optimism and religious commitment $(r=.27)$, confirming prior results.
\end{abstract}

A newly devised instrument for measuring optimism and pessimism has been described by Dember, Martin, Hummer, Howe, and Melton (in press). The original impetus for that work stemmed from an interest in investigating the correlates of individual differences in susceptibility to the Pollyanna principle-a common tendency to "accentuate the positive" that is manifested in a wide variety of circumstances (see Matlin \& Stang, 1978). Dember and Penwell (1980) reported modest correlations between one measure of the Pollyanna tendency and two indices of subjective well-being, or happiness. The measure of happiness used was chosen by virtue of its ease of administration and its good psychometric properties (Kammann, Christie, Irwin, \& Dixon, 1979); a direct measure of optimism would have been preferable, but an acceptable one was not available at the time.

The Dember et al. instrument was devised specifically to fill that gap. In the meantime, interest in optimism and pessimism burgeoned, stimulated in large part by the revised learned helplessness model of Seligman and his collaborators (e.g., Abramson, Seligman, \& Teasdale, 1978). Research performed within that framework has identified what have been called optimistic and pessimistic explanatory styles as implicated in physical health (Kamen, Rodin, \& Seligman, 1987; Peterson, Seligman, \& Vaillant, 1988) and even in the attractiveness of political candidates (Zullow, Oettingen, Peterson, \& Seligman, 1988). That work was carried out with the aid of an instrument for measuring explanatory style, the Attributional Style Questionnaire (ASQ; see Peterson et al., 1982), or with a content analysis system (Content Analysis of Verbatim Explanations, or CAVE; see Peterson, Luborsky, \& Seligman, 1983) based on the ASQ for use with existing documents.

Correspondence may be addressed to William N. Dember, Department of Psychology, University of Cincinnati, Cincinnati, OH 45221-0376.
At present, then, there are competing instruments for measuring optimism and pessimism, including a brief scale developed by Cloninger (1987) and one, very similar to ours, by Scheier and Carver (1985). It would be premature to choose among them; moreover, the various measures may prove useful for different purposes or in different contexts. With that in mind, we continue developmental research on the optimism and pessimism scale of Dember et al. (in press). In this article, we report on test-retest reliability and on correlations with the two happiness measures employed by Dember and Penwell (1980), as well as on correlations with responses to some questions modeled on items that were used in the Dember et al. study.

\section{METHOD}

\section{Subjects}

The subjects were 106 students (62 women and 44 men) from introductory psychology classes, serving for course credit. Their modal age was 19 , with a range from 18 to 30 . Ninety percent were white. The subjects were tested in groups of about 20 on each of two occasions separated by an interval of 2 weeks.

\section{Procedure}

At each testing session, each subject received a booklet containing a 60-item optimism-pessimism scale, 24 of which were filler items, with 18-item subscales measuring optimism and pessimism. The subjects rated their agreement with each item on a 4-point scale: strongly agree, agree, disagree, strongly disagree. A typical optimism item was: "I generally look at the brighter side of life." A typical pessimism item was: "I am not looking forward to the next couple of years." Prior research (Dember et al., in press) revealed that the optimism and pessimism subscales were only moderately correlated (in two studies, $r=.54$ and .57 ). Hence, the data for the present study were analyzed separately for optimism and pessimism. Following the optimism-pessimism scale were two versions of Kammann's measure of happiness. One version consisted of 12 sentences of the sort "My work gives me a lot of pleasure." The subjects responded to each sentence by choosing one of five response categories that indicated the frequency with which the subject experienced the feeling described in the sentence during the past week: not at all, occasionally, some of the time, often, all of the time. The second happiness measure consisted of 12 adjectives (e.g., "discontented") to which they 
responded in the same manner as they had responded to the sentences. Each scale yielded a single happiness measure.

Following the happiness items, the subjects were asked a series of questions, one about the likelihood of a nuclear encounter between nations and the rest about their philosophy of life and their religious and political commitments. We selected four items for correlational analysis with the optimism and pessimism measures: (1) "How likely do you think it is that there will be a nuclear encounter between nations before the year 2000?"'-to which the subjects responded on a 7-point scale from "not very likely" to "very likely"; (2) "To what extent do you have a personal philosophy of life (religious or otherwise)?" - to which they responded on a 7-point scale from "least amount" to "greatest amount"; (3) "Do you belong to, or identify with, a religious group or organization?" - to which they responded "yes"' or "no"'; and (4) "Do you belong to, or identify with, a political party, group, or philosophy?" - to which they responded "yes" or "no."

Filling out all the items in the booklet took less than an hour. Following the second session, the subjects were debriefed about the nature of the study.

\section{RESULTS}

The data permitted more analyses than we were interested in pursuing. Only those most pertinent to the purposes of this study will be reported. Of primary concern was the test-retest reliability of the optimism and pessimism subscales. For optimism, the test-retest reliability was .75 ; for pessimism, $.84(d f=104, p<.0001$, in both cases).

For purposes of comparison with previous results, the correlation between optimism and pessimism scores was .54 on the first administration and .61 on the second. The former value is remarkably close to the values (.54 and .57$)$ reported in the two studies by Dember et al., which were obtained on a single administration. The value from the second administration was slightly higher, but not significantly so.

On the first administration, optimism was significantly correlated with happiness/sentences $(r=.61)$ and with happiness/adjectives $(r=.55)$. Pessimism was also significantly correlated with happiness/sentences $(r=-.60)$ and with happiness/adjectives $(r=-.59)$. Interestingly, the two happiness measures correlated with one another only moderately more $(r=.68)$ than either did with optimism and pessimism. On the second administration, similar results were obtained between optimism and happiness/sentences $(r=.59)$ and happiness/adjectives $(r=.51)$, and between pessimism and happiness/sentences $(r=-.58)$ and happiness/adjectives $(r=-.57)$. On this administration, for the two happiness measures, $r=.76$. With regard to all of these correlations, $p<.0001$.

Correlations with the single-item variables are reported only for the first administration; second administration results show the same pattern. Neither optimism nor pessimism correlated significantly with responses to the nuclear-encounter question. Optimism was modestly predictive of a yes response to the question about having a personal philosophy of life $(r=.22, p<.03)$, but pessimism was not $(r=.16, p>.05)$. A similar and somewhat stronger result was obtained for the question about religious affiliation: Optimism was predictive of a yes response $(r=.27, p<.005)$, but pessimism scores were unrelated to this measure $(r=.003)$. Finally, the question about identification with a political group failed to yield significant correlations with either optimism or pessimism.

\section{DISCUSSION}

The primary purpose of this study was to assess the test-retest reliability of the optimism and pessimism scales. Satisfactory reliability was demonstrated for both scales, with pessimism the somewhat more reliable of the two. The latter observation is interesting in view of the fact that, in the Dember et al. (in press) study, pessimism was generally a better predictor of other measures than was optimism. In any case, these data, combined with other psychometric information reported by Dember et al., indicate that both subscales of the new instrument are psychometrically sound with regard both to internal consistency and temporal stability.

The remaining data pertain to some correlates of optimism and pessimism. In particular, we show high correlations with two (highly correlated) measures of happiness. People who report themselves to be content with their lives tend to be both high in optimism and low in pessimism. No causal implication is intended. Indeed, it may be that both an optimistic stance and expressed happiness reflect a more general tendency toward seeing things in their best light (i.e., conformity to the Pollyanna principle). Finally, we also replicated, with slightly different questions, the finding from the Dember et al. study that religious identification is associated with optimism but not with pessimism. However, we did not replicate the earlier finding of a correlation between pessimism and expectation of an imminent nuclear encounter or of a correlation between optimism and identification with a political group.

On the issue of temporal stability, we want to point out that, although our measures of optimism and pessimism have satisfactory reliability, suggesting that we are tapping a personality trait, it remains to be seen whether these measures can be modified through experimental manipulations. A study to that end is currently in progress.

\section{REFERENCES}

Abramson, L. Y., Seligman, M. E. P., \& Teasdale, J. D. (1978). Learned helplessness in people: Critique and reformulation. Journal of Abnormal Psychology, 98, 49-74.

CLONINGER, C. R. (1987). A systematic method for clinical description and classification of personality variants. Archives of General Psychiatry, 44, 573-588.

Dember, W. N., Martin, S. H., Hummer, M. K., Howe, S. R., \& Melton, R. S. (in press). The measurement of optimism and pessimism. Current Psychology.

Dember, W. N., \& Penwell, L. (1980). Happiness, depression, and the Pollyanna principle. Bulletin of the Psychonomic Society, 15 321-323.

Kamen, L. P., Rodin, J., \& Seligman, M. E. P. (1987). Explanatory style and immune function. Unpublished manuscript, University of Pennsylvania, Philadelphia, PA.

Kammann, R., Christie, D., Irwin, R., \& Dixon, G. (1979). Properties of an inventory to measure happiness (and psychological health). New Zealand Psychologist, 8, 1-12.

Matun, M. W., \& Stang, D. J. (1978). The Pollyanna principle. Cambridge, MA: Schenkman.

Peterson, C., Luborsky, L., \& Seligman, M. E. P. (1983). Attributions and depressive mood shifts: A case study using the symptomcontext method. Journal of Abnormal Psychology, 92, 96-103.

Peterson, C., Seligman, M. E. P., \& Vaillant, G. E. (1988). Pessimistic explanatory style as a risk factor for physical illness: A thirtyfive-year longitudinal study. Journal of Personality \& Social Psychology, 55, 23-27.

Peterson, C., Semmel, A., von Bayer, C., Abramson, L. Y., MetalSKY, G. I., \& Seligman, M. E. P. (1982). The Attributional Style Questionnaire. Cognitive Therapy \& Research, 6, 287-299.

SCHEIER, M. F., \& CARVER, C. S. (1985). Optimism, coping, and health: Assessment and implications of generalized outcome expectancies. Health Psychology, 4, 219-247.

Zullow, H. M., Oettingen, G., Peterson, C., \& Seligman, M. E. P. (1988). Pessimistic explanatory style in the historical record: CAVing LBJ, presidential candidates, and East versus West Berlin. American Psychologist, 43, 673-682. 